\title{
Design challenges for matrix assisted pulsed laser evaporation and infrared resonant laser evaporation equipment
}

\author{
James A. Greer
}

Received: 6 July 2011 / Accepted: 20 August 2011 / Published online: 12 October 2011

(c) The Author(s) 2011. This article is published with open access at Springerlink.com

\begin{abstract}
Since the development of the Matrix Assisted Pulsed Laser Evaporation (MAPLE) process by the Naval Research Laboratory (NRL) in the late 1990s, MAPLE has become an active area of research for the deposition of a variety of polymer, biological, and organic thin films. As is often the case with advancements in thin-film deposition techniques new technology sometimes evolves by making minor or major adjustments to existing deposition process equipment and techniques. This is usually the quickest and least expensive way to try out new ideas and to "push the envelope" in order to obtain new and unique scientific results as quickly as possible. This process of "tweaking" current equipment usually works to some degree, but once the new process is further refined overall designs for a new deposition tool based on the critical attributes of the new process typically help capitalize more fully on the all the salient features of the new and improved process. This certainly has been true for the MAPLE process.

In fact the first MAPLE experiments the polymer/solvent matrix was mixed and poured into a copper holder held at $\mathrm{LN}_{2}$ temperature on a laboratory counter top. The holder was then quickly placed onto a $\mathrm{LN}_{2}$ cooled reservoir in a vacuum deposition chamber and placed in a vertical position on a $\mathrm{LN}_{2}$ cooled stage and pumped down as quickly as possible. If the sample was not placed into the chamber quickly enough the frozen matrix would melt and drip into the bottom of the chamber onto the chambers main gate valve making a bit of a mess. However, skilled and motivated scientists usually worked quickly enough to make this process work most of the time. The initial results from these
\end{abstract}

J.A. Greer ( $₫)$

PVD Products, Inc, Wilmington, MA, USA

e-mail: jgreer@pvdproducts.com experiments were encouraging and led to several publications which sparked considerable interest in this newly developed technique

Clearly this approach provided the vision that MAPLE was a viable deposition process, but the equipment was not optimal for conducting MAPLE experiments on a regular basis for several reasons. The first reason is that the polymer/solvent mix as well as the sample holder are both exposed to the humidity in the air which will coat the entire surface of the holder and target with water vapor. Some polymer and/or solvent materials may not react well with water vapor. Also, the layer of water vapor absorbed on the target surface may then absorb the incident laser radiation until it is removed from the surface. Thus, it may be unclear when the water vapor is fully removed from the polymer/solvent surface and the MAPLE deposition process actually occurs. This makes deposition of specific polymer thickness difficult to calculate. While it is well known that Quartz crystal microbalances do not work well for PLD of oxide materials it can be used for the deposition of MAPLE materials. However, with rastered laser beams the tooling factor becomes a dynamic number making interpretation of final thickness potentially difficult without careful precalibration.

Another serious issue with the initial MAPLE process was related to the use of UV lasers such as an excimer operating at 193- or 248-nm or frequency tripled, Nd:YAG lasers at $355 \mathrm{~nm}$. These lasers have high energy per photon (between about 6.4 to $3.5 \mathrm{eV}$ ) which can lead to a variety of deleterious photochemical mechanisms that can damage the polymer chains or organic structure. Such mechanisms can be direct photo-decomposition by photochemical bond breaking and photothermal effects. Alternative lasers, such as a Er:YAG laser operating at 2.9 microns produce photons with energy of $\sim 0.43 \mathrm{eV}$. Such longer wavelength lasers 
have been used for the IR-MAPLE process and may be very useful for future MAPLE systems.

A third issue with the initial approach to MAPLE was that the process did not lend itself easily to growing multilayer films. Most standard pulsed laser deposition tools have "multi-target" carousels that allow for easy target changes and multilayer film growth. This is true for sputtering, MBE, and evaporation equipment as well. This multilayer feature would certainly benefit the MAPLE process for the growth of multilayer organic materials.

Another more recent advancement in thin-film laser deposition is that of Resonant Infra Red Pulsed Laser Deposition (RIRPLD) of polymer materials. This process is more akin to standard PLD but uses tunable lasers with which to select the proper wavelength to couple to vibration bands of a solid polymer, or in some cases a polymer/solvent MAPLE mixture. This technique was developed under a collaboration of researchers at the Naval Research Labs and the Free Electron Laser (FEL) at Vanderbilt University. The wide tuning range of the FEL and its relatively high power make it a very attractive source for RIRPLD. However, the price of such lasers - of order several million dollars in capital costs alone-is very high and well beyond the budgets of most research institutions. Advances in RIRPLD are currently limited due to the scarcity of tunable lasers with sufficient power in the IR range of interest to obtain reasonable deposition rates.

Over the past nine years commercial equipment for MAPLE has been on the market and new lasers are being developed that may significantly improve MAPLE and RIRPLD capabilities. Examples of basic single-target MAPLE equipment, as well as multiple target MAPLE systems are described. Discussion of current lasers for MAPLE and RIRPLD are given. Finally, even though these processes have been around for a significant amount of time there are still many unknowns associated with these techniques that still should be explored before these processes can be used for production of useful products. Some of these issues which need to be addressed will be discussed.

\section{Introduction}

The MAPLE and RIRPLD processes are still relatively new and fertile ground for technology and equipment development. PLD became popular in the mid 1980s and many researchers started to get involved in utilizing the PLD process mostly with in-house modified deposition equipment to grow a wide variety of non-organic thin films [1, 2]. Within a few years several companies were created to meet the demanding needs of customers who wanted off-the-shelf equipment for PLD applications. Today, a large fraction of
PLD equipment is now purchased from a number of such vendors. MAPLE and RIRPLD on the other hand have been around for about 13 and 12 years, respectively [3-5]. While MAPLE and RIRPLD have been around for some time their popularity has not grown as fast as that of standard PLD most likely for several reasons. First, it is not straight forward to modify existing PLD equipment to make a MAPLE system and second, the types of laser used in these deposition processes are not readily available. Also, most researchers initially were more focused on inorganic material film growth. However, the interest in organic thin films has grown significantly for a wide array of applications over the past 10 years so there is a strong need for development of equipment to meet the needs of current and emerging organic thin-film growth. Commercial equipment is slowly coming on-line that can address some of the issues discussed above. However, there are still a lot of unknowns about the intricacies of these processes and it will take considerable more time in order to gain a full understanding of the processes as well as to realize equipment that is fully functional to meet all the needs of current organic thin-film research.

\section{MAPLE Deposition Equipment}

As noted above, the first MAPLE deposition tools were basically clever modifications to existing standard PLD tools $[3,4]$. While the concept worked and proved the basic principles of the MAPLE process, these tools were not ideal for making MAPLE films on a regular basis. Clearly with a liquid target that will eventually become frozen, the target source should be positioned on the bottom of the chamber where gravity holds the liquid and the Polymer/Solvent $(\mathrm{P} / \mathrm{S})$ mix can be frozen in place. Also, upon heating up the sample when positioned properly the liquid will not drop into the base of the chamber potentially contaminating future films with different solvents and organic materials.

The choice of the polymer and solvent mix are very critical for MAPLE deposition. The ultimate goal is to find a solvent that is volatile enough not to become incorporated in the thin film (i.e. it should be pumped away after evaporation), is capable of diluting the polymer material to concentrations from say $0.1 \%$ to about $5 \%$, and will freeze solid at temperatures above that of liquid nitrogen $(77 \mathrm{~K})$. Another strong feature of the solvent is its absorption coefficient with respect to the laser radiation being used. Strong coupling will provide for better laser coupling to the solvent and deposition rates.

Figure 1 shows a photograph of a commercially available MAPLE system. This system uses a single rotating MAPLE target assembly housed in a $\mathrm{LN}_{2}$ reservoir located on the 
Fig. 1 Photograph of a commercial MAPLE deposition system using a 1.5 watt Er:YAG laser

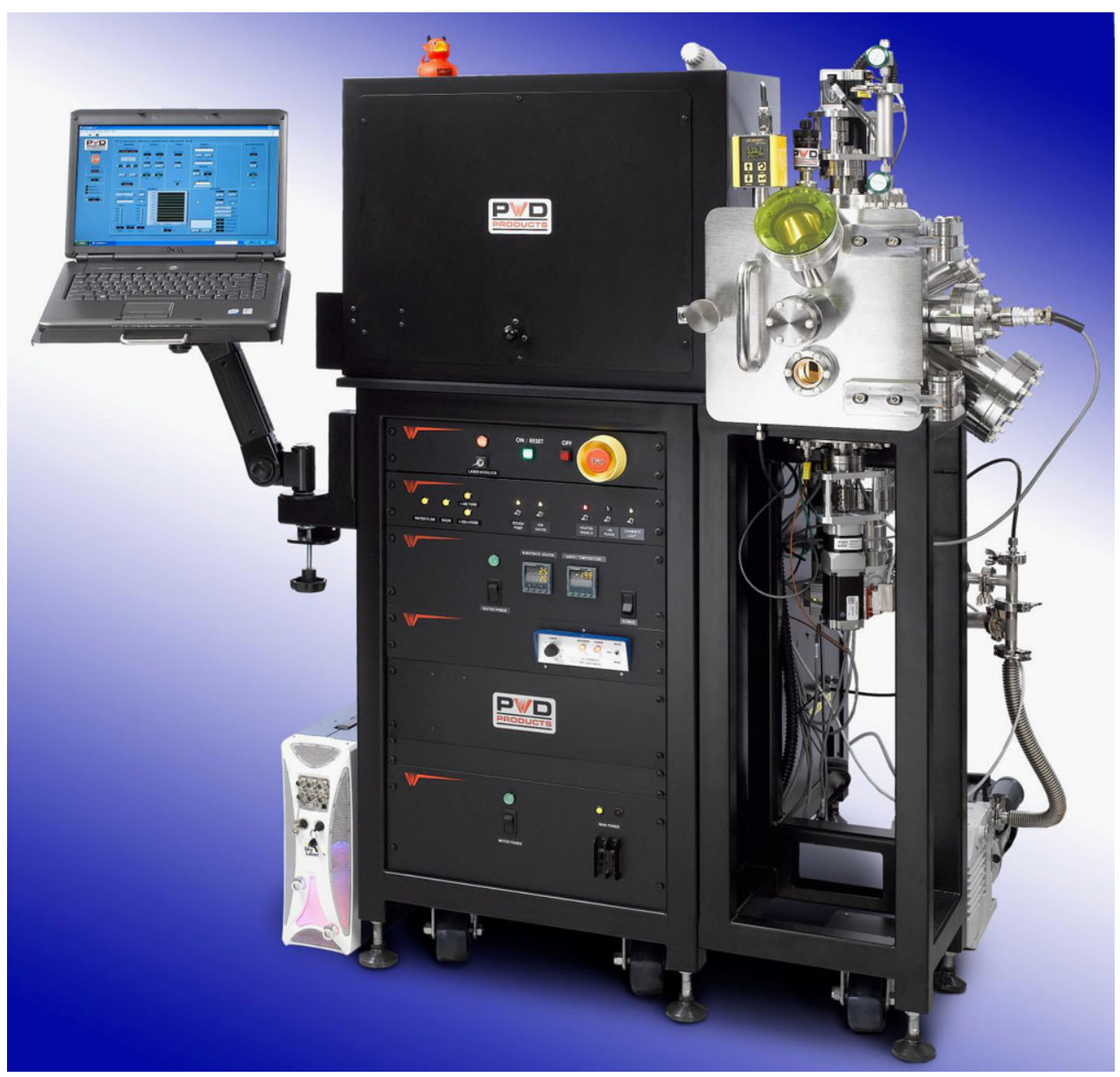

bottom of the chamber as seen in Fig. 2. The substrate is located directly above the target and the target-to-substrate distance can be adjusted from about 4 to $10 \mathrm{~cm}$ (in this case manually). Substrates can easily be inserted in place through the chambers front door, or via an optional loadlock. Just like in regular PLD, the substrate is typically rotated at rates up to about 40 RPM and the laser beam is rastered across the rotating target to make efficient use of target material and provide more uniform film coatings [2, p. 191]. The MAPLE target is also rotated at rates of about 10 RPM. Faster target rotation is somewhat difficult as one must provide good thermal contact with the $\mathrm{LN}_{2}$ reservoir to keep the sample frozen. Vacuum chamber pumping packages would typically include a turbo pump and oil based mechanical pump. In some cases corrosive series turbo pumps may be necessary based on the solvent materials being utilized. Cryo pumps would be a very poor choice for this process due to the collection of large amounts of solvent that would be trapped in the pump body. In most cases dry pumps do not react well to pumping large amounts of organic solvent materials. In order to control the energetics and plume shape of the ejected material the chamber can be back filled with argon (or other) gas via an MFC. Closed-loop pressure control is also possi- ble using a capacitance manometer and stepper motor controlled gate valve. Standard vacuum gauging such as an ion and convectron gauges can be used for most materials.

The laser used in the system shown in Fig. 1 was an Er:YAG laser with output at 2.9 microns and maximum output of $150 \mathrm{~mJ}$ per pulse, $10 \mathrm{~Hz}$ rep rate, and a $100-150 \mathrm{mi}-$ crosecond pulse length with the laser in free-running mode. The laser is housed within the large black box mounted on top of the electronics rack. The housing provides a laser safe enclosure with removable panel. The panel is interlocked with the laser to shut it down when removed. This provides safe access to the laser and optical components. Also, all viewports include an optical filter to protect the viewer's eyes from reflected laser radiation. One issue to deal with at this wavelength is that water vapor absorbs the 2.9-micron radiation and thus the optical train should be kept as short as possible or purged with dry nitrogen. Nd:YAG lasers or other lasers can be used as well. But as noted above, the longer the wavelength typically the better the quality of polymer film deposited [2, p. 63]. Optical components that are typically used are $\mathrm{CaF}_{2}$ or $\mathrm{BaF}_{2}$ for both the focus lens and chamber entrance window. AR coated silver mirrors are used as beam turning elements for this wavelength. As men- 
Fig. 2 Photograph of the inside of a single target MAPLE deposition system

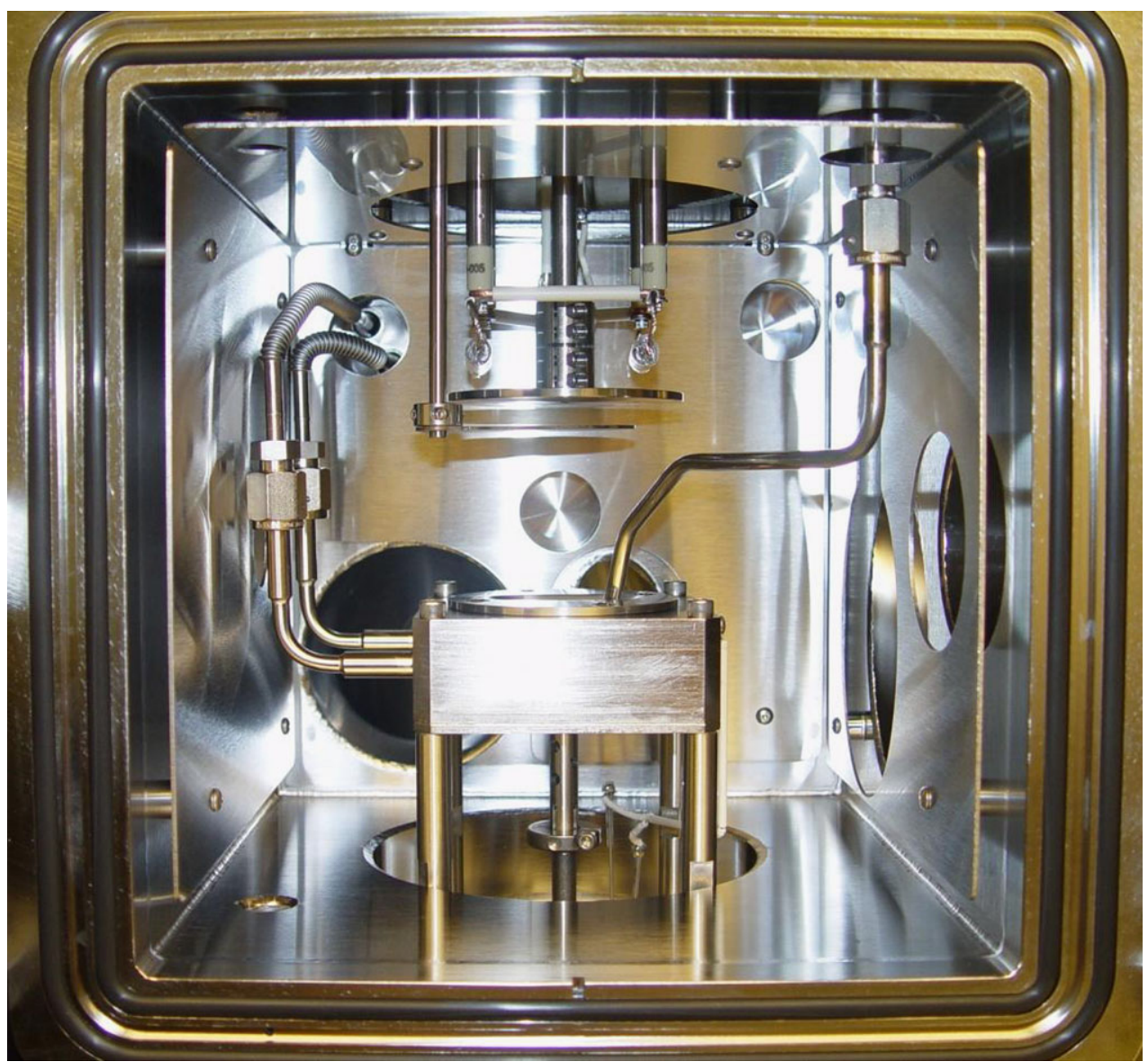

tioned above, one of the mirrors would be mounted to a kinematic mirror mount with computer controlled linear actuator to raster the laser beam across the MAPLE target surface to enhance film uniformity [2, p. 191]. One problem typically encountered in standard inorganic PLD is the slow but steady coating of the laser entrance window. This coating can significantly reduce the energy density of the target and affect deposition rate and film properties [2, p. 191]. This type of problem is not expected to be serious in the MAPLE process as it is expected that the laser radiation is sufficient to keep the laser entrance window clean of polymer buildup.

As mentioned above freezing the target in an atmosphere of air is not a good idea as it leads to adsorption of water vapor on the target surface. In order to deal with the issue of contamination via condensed water vapor it is best to first pump the vacuum chamber down to its base pressure, the lower the better. Typical systems can be pumped to the low $10^{-7}$ or $10^{-8}$ Torr range with loadlocks. Then the chamber can be back filled with dry nitrogen, argon or other dry or inert gas to a pressure just below that of atmosphere. Injection of the $\mathrm{P} / \mathrm{S}$ mix at this point will minimize interaction with water vapor. Figure 3 shows a scheme that allows the chamber to be pumped and the polymer/solvent mix to be inserted into the target cup and then frozen in place. In this case, a fixed amount of P/S mix (about $20 \mathrm{cc}$ ) is placed into a separate "Fill Tube" (FT). The FT has a removable flange with an NW fitting on top that can be removed to fill it with the P/S liquid mix with the ability to be purged with argon or dry nitrogen once the top flange fitting is put back in place. When the main chamber has been properly pumped and back filled as described above to a pressure of say 650-700 Torr so the main chamber door does not open, a valve between the FT and chamber can be opened. The pressure difference between the FT chamber and main chamber pushes the liquid P/S mix through a directional tube directly into the target cup holder within the chamber as seen in Figs. 2 and 3. At this point $\mathrm{LN}_{2}$ can be injected into the reservoir that surrounds the target cup assembly and the polymer/solvent mix can be frozen in place at a relatively high pressure. If one starts to pump on the system prior to freezing a good amount of the solvent may be lost and the concentration of the polymer/solvent mix will be altered. Once the P/S mix is frozen the system can be pumped down to its base pressure. The base pressure reached will depend strongly on the vapor pressure of the P/S mix selected at the temperature of the reservoir. Typical reservoir temperatures are about -170 to $-195^{\circ} \mathrm{C}$ once fully cooled down. It should also be pointed out that even when pumping out the chamber to its base pres- 
Fig. 3 Schematic of Fill Tube (FT) for P/S injection into the main deposition chamber

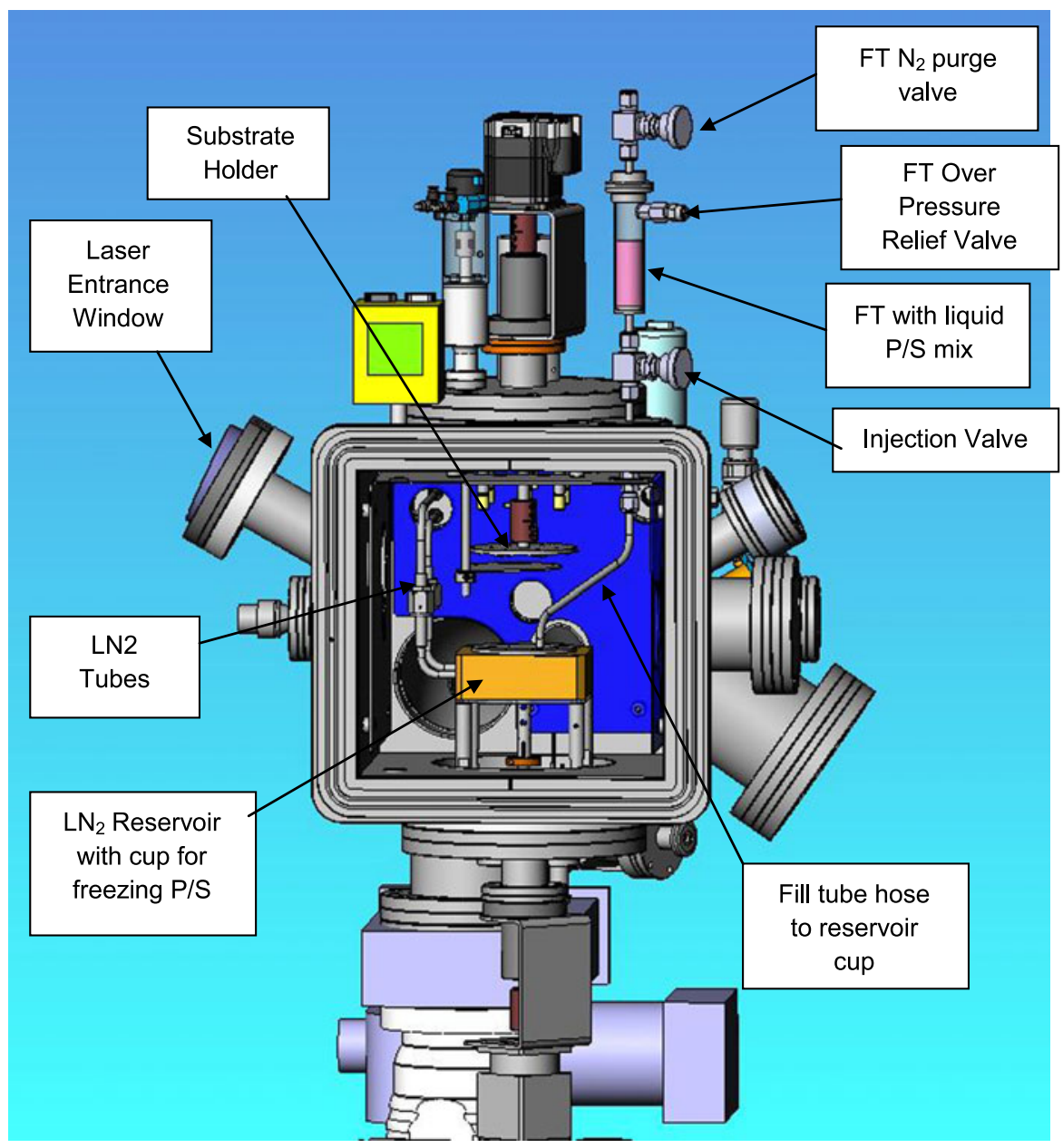

sure of say $5 \times 10^{-7}$ Torr, the chamber will still have considerable amount of water vapor adhered to the walls. It is well known that water vapor is the dominant gas specie in a properly pumped SS chamber in this pressure range. As a rule of thumb, a monolayer per second of gas arrives at the surface of a sample at a pressure of about $2 \times 10^{-6}$ Torr. Thus, all frozen MAPLE targets will have some degree of water vapor on the surface. At this point the process of deposition can start. The chamber can be back filled with an inert gas to the desired pressure if required and the laser can be fired for the desired length of time. Film growth will depend strongly on the concentration of polymer in the P/S mix, the chamber pressure, target-to-substrate distance, solvent absorption to the laser radiation, and overall laser energy on target.

The system in Fig. 1 does not include a loadlock. However, the addition of a loadlock is well worth the expense for MAPLE deposition systems. Besides increasing the overall throughput of a system a loadlock for a MAPLE tool will allow several films to be grown from the same P/S mix sample. In a system without a loadlock the $\mathrm{LN}_{2}$ reservoir will need to be heated up to room temperature to open the chamber and change the substrate sample. Once the reservoir is heated up it is typically necessary to remove the remaining $\mathrm{P} / \mathrm{S}$ mix and start with a fresh mixture. In some cases the materials used in the P/S mixes can be rather expensive and this constant recharging can become quite costly. In such cases it is possible to reduce the volume of the target holder cup.

One interesting variation of the use of a loadlock in a single-target MAPLE system is to facilitate the growth of multilayers without exposing the sample to atmosphere [6]. In this case, after the first layer of polymer film was deposited the sample was extracted from the main chamber and stored in the loadlock and kept under high vacuum conditions ( $\sim 10^{-7}$ Torr). The MAPLE target was then heated to room temperature and the chamber opened and the remaining $\mathrm{P} / \mathrm{S}$ mix was removed from the target cup. A new $\mathrm{P} / \mathrm{S}$ mix was then injected into the system and the target was then frozen by injection of $\mathrm{LN}_{2}$ into the reservoir. The chamber was then pumped down to its base pressure. At this point the wafer held within the loadlock was then re-inserted onto the substrate stage and a second polymer layer was deposited. In principle, this process could be repeated multiple times. However, it is slow and cumbersome. One also needs to consider a technique to heat up the $\mathrm{LN}_{2}$ reservoir quickly once 
Fig. 4 Photograph of the large area multi-target MAPLE/RIRPLD system installed at Jefferson Laboratories, Newport News Virginia. Photo courtesy of Jefferson Laboratory

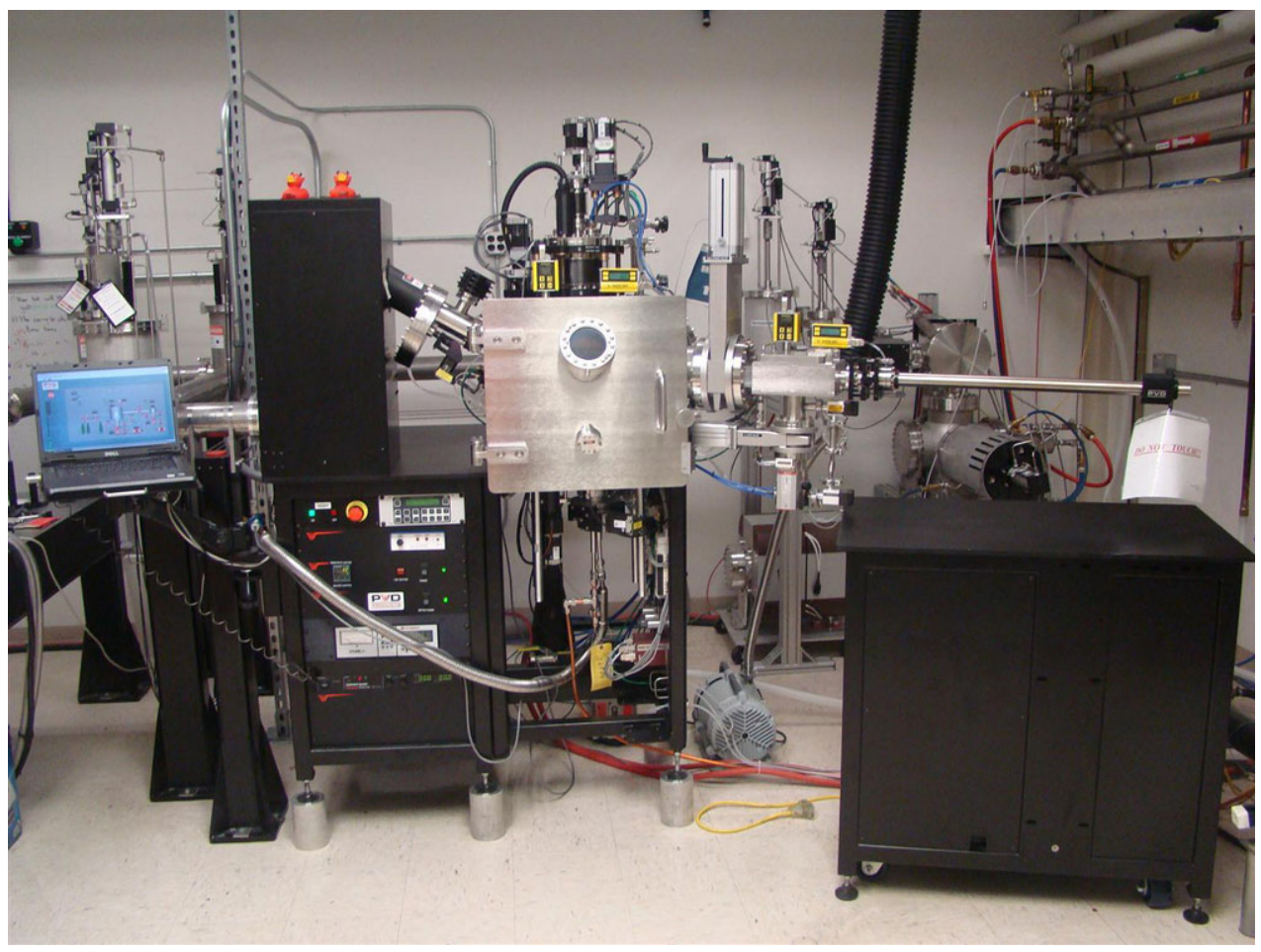

deposition is complete. Preheated nitrogen can be injected into the reservoir using a proper set of valves for this purpose.

An alternative approach to multilayer polymer film growth is to use a system that incorporates multiple MAPLE targets within the chamber at one time. Such a system is described below.

The system seen in Fig. 1 uses a single MAPLE target, and thus, one polymer material can be deposited at one time. In some cases it is desirable to deposit multiple films in order to obtain specific desired properties. This creates a considerable design challenge for the system manufacturer since multiple rotating MAPLE targets must be provided.

Such a system has been created and shipped to the Free Electron Laser Facility at Jefferson Laboratory in Newport News Virginia in late 2007. Figure 4 shows a photograph of the system installed in the laboratory. This system is designed to work in the visible and IR region of the FEL. It includes a target manipulator that has six targets all 2-inch in diameter, three MAPLE targets and slots for three solid targets. The system includes three separate fill tubes for the injection of three different $\mathrm{P} / \mathrm{S}$ mixes. The solid targets could be either organic or inorganic materials. All of the targets are held within a large liquid nitrogen reservoir. With this suite of targets the system can be used for both MAPLE and RIRPLD, or a combination of both making stacks of polymer or polymer/inorganic multilayers. The system includes a lamp based substrate heater assembly with the ability to heat substrates to $500^{\circ} \mathrm{C}$. The heater was integrated with a large bore programmable Z-stage that provides a variable target-to-substrate distance ranging from 60 to $125 \mathrm{~mm}$. The system includes a loadlock for quick wafer transfers, closedloop pressure control using a capacitance manometer and stepper motor controlled gate valve, a 1,200 1/s turbo pump, and two MFC's calibrated for oxygen and argon. In order to deal with buildup of inorganic material on the laser entrance window the system also included a large Intelligent Window. This window helps keep the beam path clean for extended periods of time and also allows the energy that is about to hit the target be sampled when desired [2, p. 191]. Since the system is mated to a laser with a highly focused laser beam the optical train included the ability to move the focus lens with the laser raster mirror used to scroll the laser across the ablation target [2, p. 191]. In this way the spot size and laser fluence can be kept constant during the raster process. The system is fully computer controlled to provide the ability to grow multilayer films.

Figure 5 shows a photograph of the internal components of this system during fabrication. The gold coated substrate heater assembly is located above the target manipulator assembly. A shutter is provided for pre-ablation of the target surface. The target manipulator sits in the lower section of the chamber and has a large stainless steel shield above it to protect targets from cross contamination and also to minimize the affect of substrate heating on the $\mathrm{LN}_{2}$ reservoir. A slot seen on the left side of the stainless shield is where the laser radiation hits the active ablation target. Figure 6 shows the target manipulator assembly with the stainless 
Fig. 5 Photograph of the inside of the Jefferson Lab large-area MAPLE RIRPLD deposition tool. The substrate heater is housed within the gold coated block on the top of the chamber and the MAPLE target manipulator sits underneath the SS plate. The internal Swagelok connections for the three fill tubes can be seen in the upper right hand corner of the chamber

Fig. 6 Photograph of the multi-target $\mathrm{LN}_{2}$ cooled manipulator assembly used in the Jefferson Labs

MAPLE/RIRPLD system with the large stainless top plate removed. The three fill tubes for three MAPLE P/S mixes are seen on sitting above the targets in the upper right of the photo
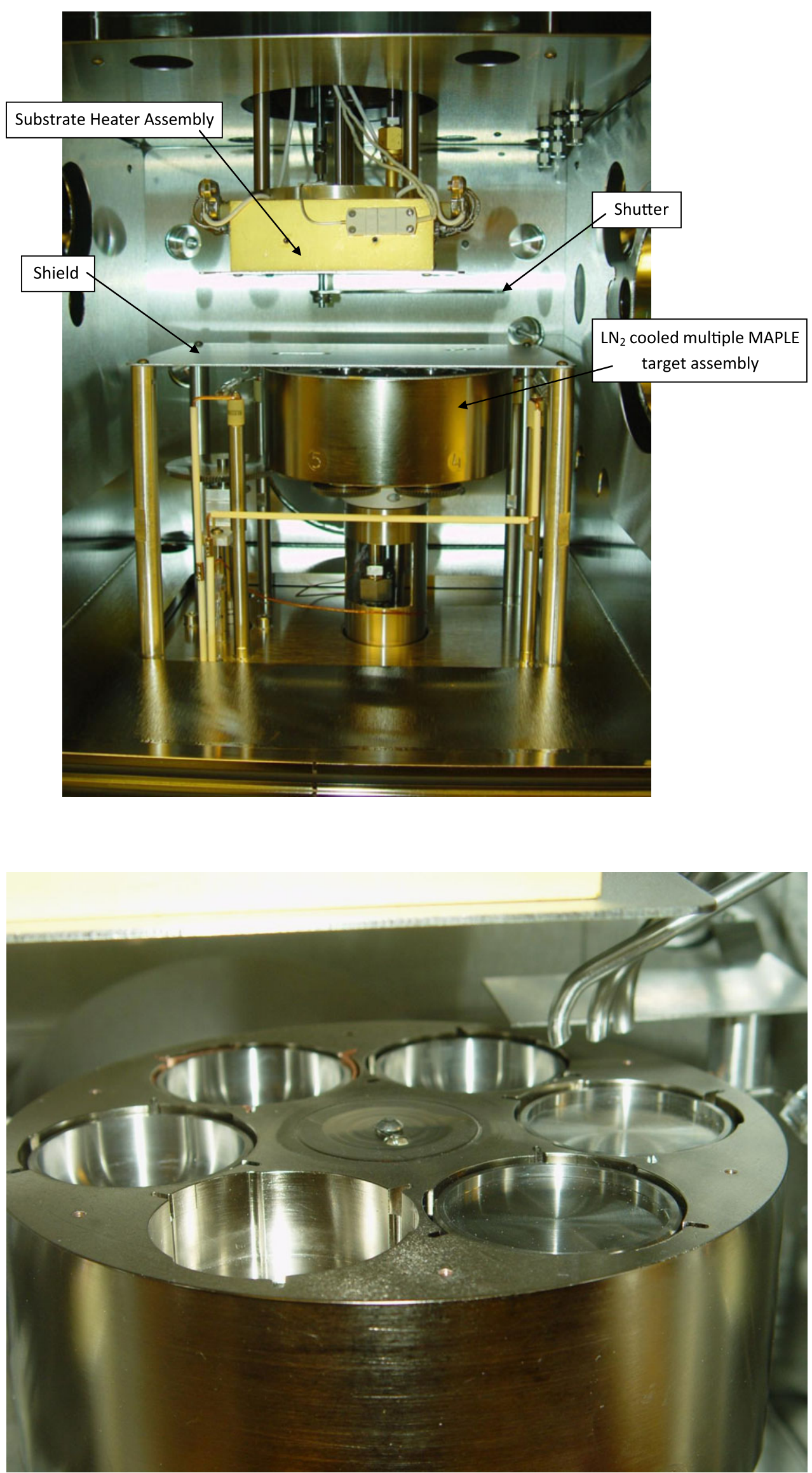
Fig. 7 Schematic of a large area MAPLE system with cutout in the shutter to act as a mask. The Wafer sits directly behind the shutter surface

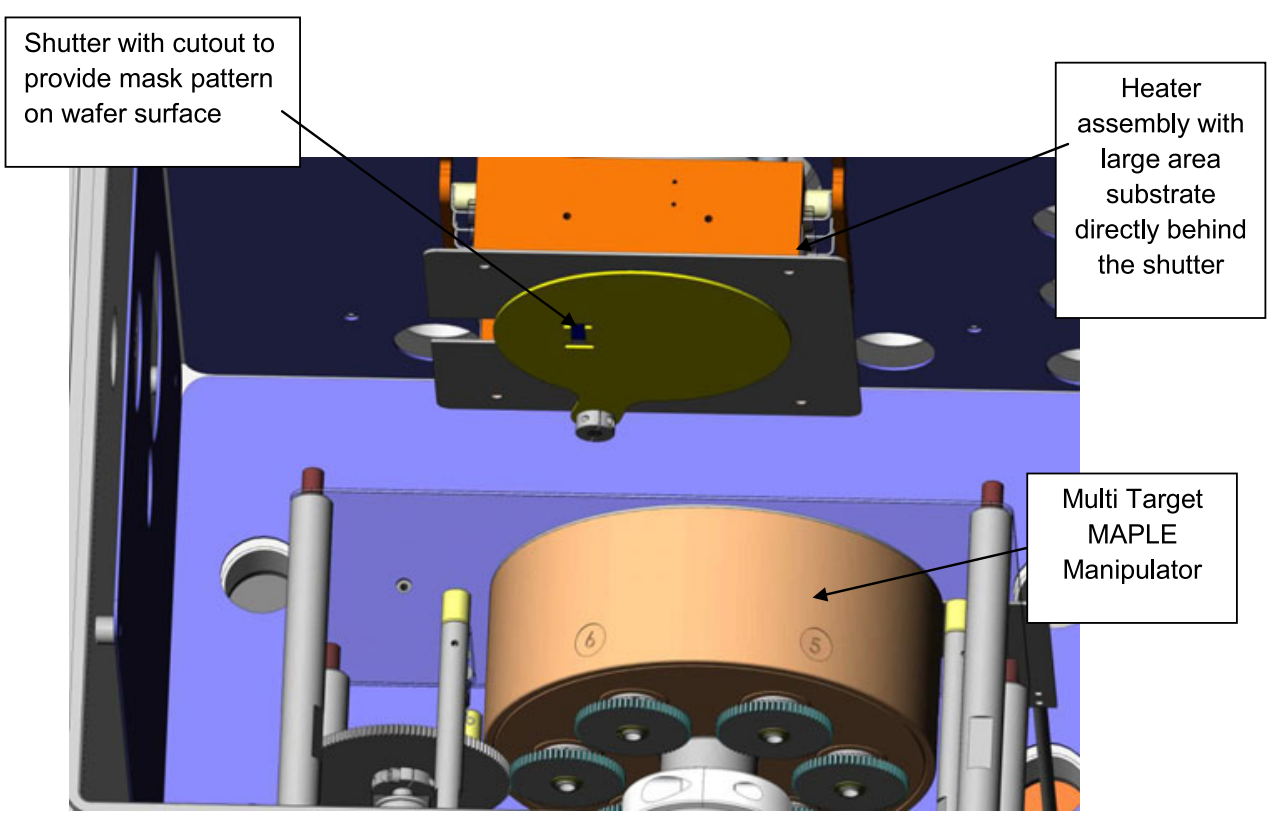

steel shield removed. Three cupped MAPLE targets can be seen along with two of the three pedestals for solid targets. The overall housing is made from a machined OFHC copper block that has been brazed together and can readily be filled with $\mathrm{LN}_{2}$ from a central shaft through the bottom of the assembly. The system can rotate up to $360^{\circ}$, and each target can rotate around its own central axis continuously when indexed into the deposition position (below the laser beam). Three SS tubes are seen on the right hand side of the photo. These are connected to three separate MAPLE target fill tubes, one each for the MAPLE pedestals.

One aspect of growing thin films (organic and inorganic) is to deposit patterned structures for various applications. Patterned films can be deposited by direct-write processes such as Laser Induced Forward Transfer or LIFT. As an example of the LIFT process that is in production precise frequency trimming of SAW devices has been demonstrated for use in radar and communication systems [7]. A more basic way of making patterned films for R\&D is by using meta masks to define an area on the substrate surface that you want to coat. Using masks in the proper way allows one to deposit a number of patterns on a single wafer to conduct parametric studies of laser power, multilayer materials, combinatorial films, etc. Figure 7 shows a schematic of large area MAPLE system with a cutout in the shutter which is acting as a mask. The wafer sits within a few $\mathrm{mm}$ of the mask surface. If the wafer is kept fixed in position (not rotating) for the deposition the pattern of material will be directly transferred to the substrate surface. After the deposition is complete, the wafer can be indexed a fixed amount and then a new film deposited with different parameters or different materials can be deposited. In this way one can make a wafer with multiple patterns with features down below $1 \mathrm{~mm}$, each pattern deposited under different conditions. Figure 8 shows a schematic of examples of such patterns using the mask assembly in Fig. 7.

While MAPLE has been used for the growth of organic and biological materials for several years there are still several outstanding questions remaining about the process. These include the following.

1. What is the angular distribution of the plume produced in the MAPLE process? How does this depend on laser wavelength, pulse length, spot size, angle of incidence, and chamber background pressure?

2. What types of film thickness uniformity can be produced over large substrate sizes with the MAPLE process?

3. Once the polymer/solvent mix has been frozen in place, what is the distribution of the polymer dispersed throughout the frozen target? Is there a concentration gradient within the $\mathrm{P} / \mathrm{S}$ target?

4. What is the best pulse length and wavelength to use for this process for different solvent and polymer materials?

5. What effect does absorbed water vapor play on the laser interaction with the target?

One significant issue faced by the manufacturer of such equipment is that these questions cannot typically be answered before the tool must be shipped. This leaves answers to these questions with the customers in the research community. However, the customers are more focused on exploring the more fundamental material properties of MAPLE films than that of answering some of the more mundane questions noted above. The last question above has been addressed in an interesting paper by Pate and Stiff-Roberts [8]. 
Fig. 8 Schematic of four films patterns deposited via the MAPLE Process. Each pattern could represent a different film deposited using different deposition parameters such as laser fluence, laser wavelength, or films grown as multilayers with various film thicknesses, etc.

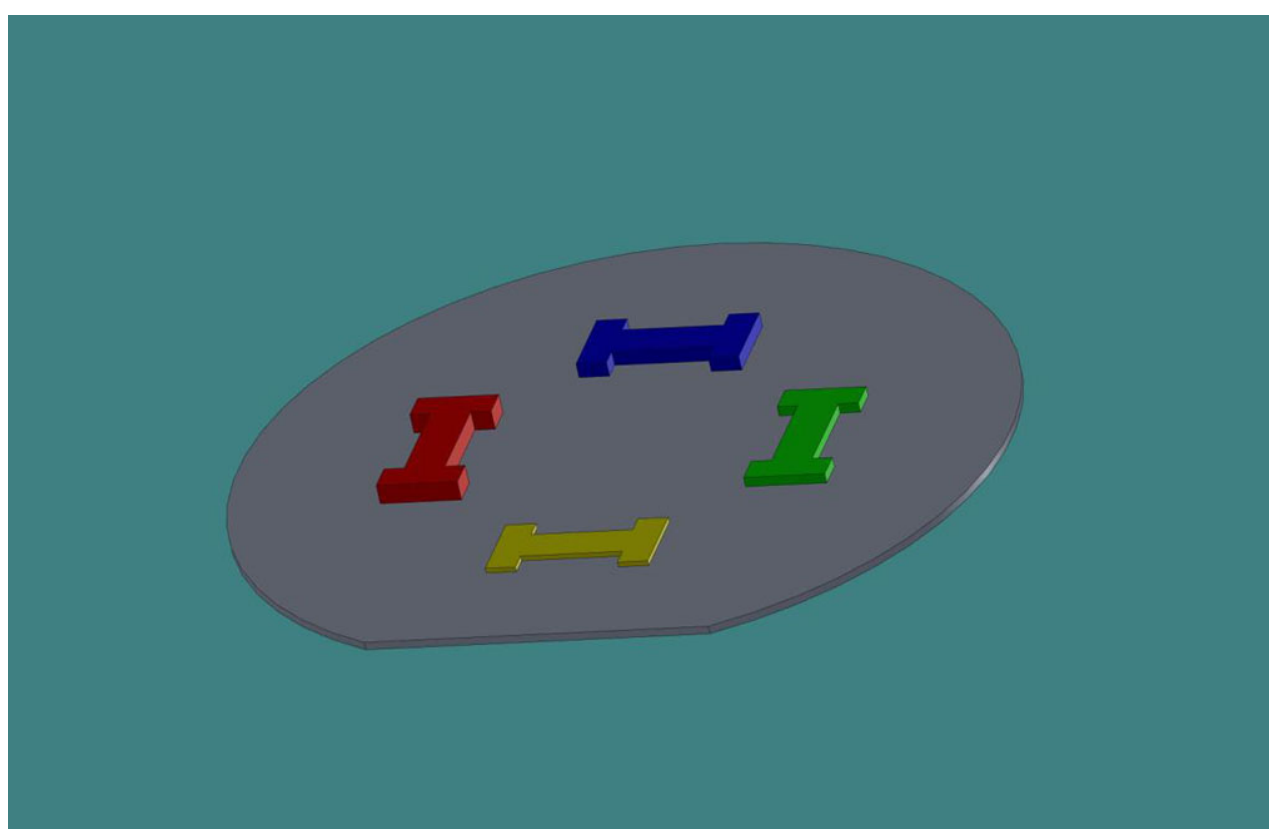

\section{Resonant infrared pulsed laser deposition}

Resonant Infrared PLD was first described by Bubb et al. [5]. This unique deposition approach uses the tunability of a laser to couple to vibrational bands of an organic material such as a polymer. In some cases the coupling at one vibrational band is strong and good transfer for solid material into thin film is obtained. At other vibrational bands coupling can be strong but localized heating can damage the target and yield very poor film deposits. This approach to laser deposition has the potential to deposit an enormous amount of organic material in thin-film form if the proper laser can be obtained. To date, RIRPLD has relied mostly on Free Electron Lasers which are extremely expensive and unfortunately not readily available to most researchers.

What is ideally needed for this process to become more readily available to researchers is a tunable laser in the mid IR region. The ideal laser would have a variable pulse length in the 10-50 picoseconds range, with a tuning range between about say 2.5 to 5 microns. Longer pulse lengths tend to boil the polymer target and create a heat-affected zone. Energy per pulse should be on the order of $10 \mathrm{~mJ}$ to $100 \mathrm{~mJ}$ and repetition rates variable from a few $\mathrm{Hz}$ to say $1,000 \mathrm{~Hz}$. While no laser of this type is presently commercially available, a prototype has been constructed that successfully produced resonant IR ablation of polystyrene with tunable light around 3.4 microns. The prototype was constructed from commercially available components and could thus be both replicated and improved without going to custom components. That said, however, depositing organic and polymeric films based on these processes will move along slowly until new lasers with improved properties can be established and become commercially available.
Recently a tunable IR laser prototype suitable for polymer ablation has been demonstrated based on a Nd:YAG pump laser coupled to an optical parametric amplifier [9]. This laser has a 8 ps pulse duration with between $2 \mathrm{~mJ}$ per pulse at the pump wavelength of $1064 \mathrm{~nm}$ and a maximum $10 \mathrm{~Hz}$ repetition rate. This provided $170 \mu \mathrm{J}$ per pulse of tunable IR light and about $2 \mathrm{~mW}$ of average power. This new laser is based on a two-stage seeded optical parametric amplifier with a tuning range of 2.9 to 3.6 microns. This is an excellent tuning range for depositing many polymer materials via RIRPLD as well as MAPLE. Moreover, this prototype was built entirely from commercial components, suggesting the possibility for substantial improvements in the future.

Designs are presently being considered for all solid-state tunable IR systems, based on 10-50 ps pump Yb:YAG or $\mathrm{Nd}: \mathrm{YVO}_{4}$ pump lasers that can operate at pulse repetition rates up to $1 \mathrm{kHz}$ with average powers of $10 \mathrm{~W}$. A two-stage optical parametric amplifier using periodically poled lithium niobate (PPLN) frequency conversion crystals, seeded by a commercial erbium-doped fiber amplifier (EDFA) used in telecommunications systems, is expected to yield a tunable IR average power around $1 \mathrm{~W}$ with $1 \mathrm{~mJ} /$ pulse. This set of specifications would in fact be viable at least at the deviceprototyping level. In fact, one has to be careful how much power one dumps into a MAPLE target as it can cause localized melting of the frozen P/S target material. The price of such a laser is expected to be about 1.5 times that of an 80 watt excimer laser. It is expected that this laser would have high enough frequency conversion efficiency so that it could also potentially produce UV radiation for inorganic PLD with the fourth harmonic of the pump laser. Thus, it 
Fig. 9 Photograph of a MAPLE/RIRPLD tool with loadlock for 2-inch wafers. The system uses a frequency doubled Continuum PowerLite Nd:YAG laser and Surelite OPO Plus. The tunable range of the system ranges from about 680 $\mathrm{nm}$ to $2,500 \mathrm{~nm}$ with power ranging from about 1.2 watts in the visible to less than 0.2 watts at $2,500 \mathrm{~nm}$

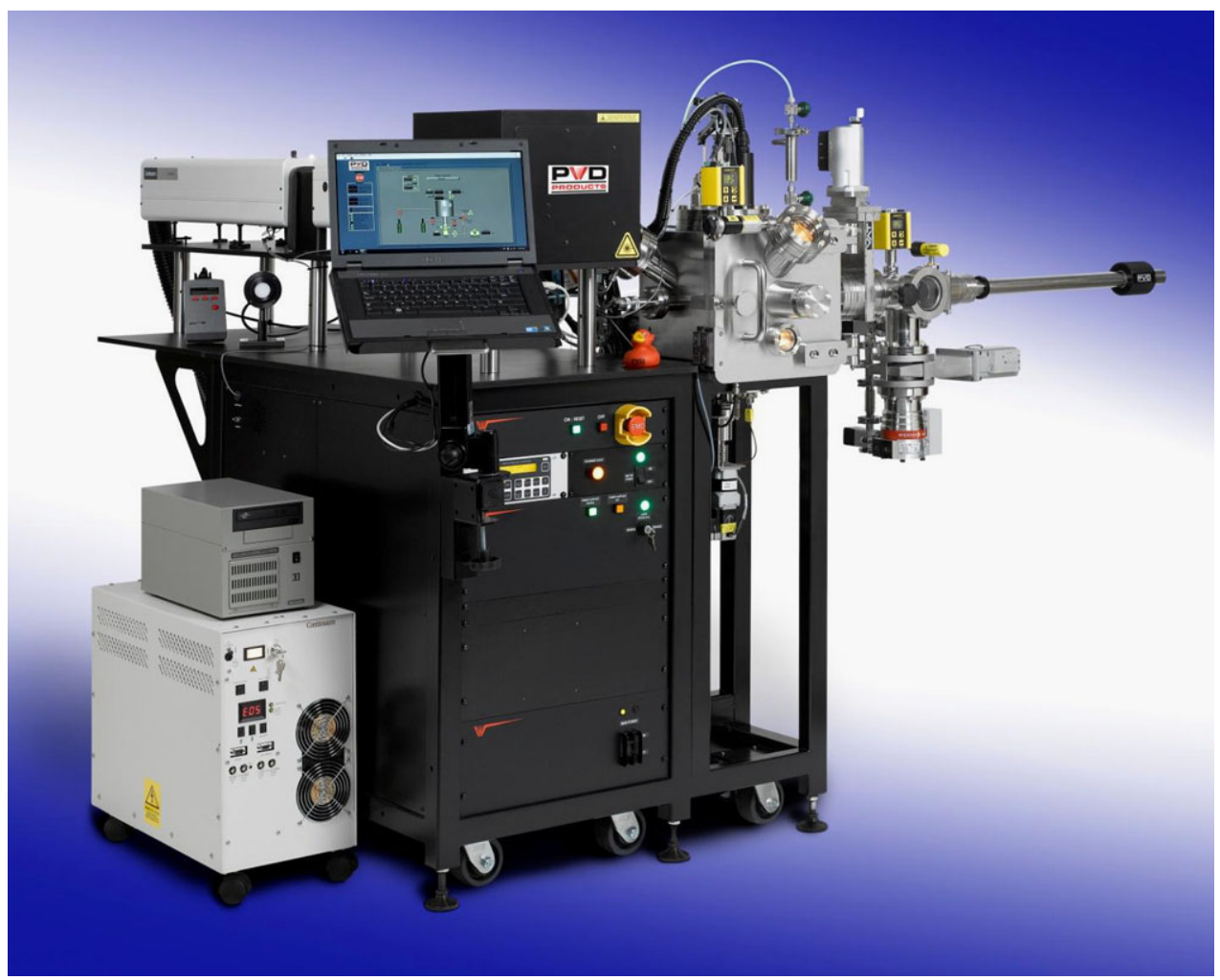

may ultimately be used for both organic and inorganic PLD justifying to some extent its high initial cost.

At the present time there are no commercial lasers available that are tunable within the mid IR band of interest with energies required. Q switched Nd:YAG lasers can be frequency doubled $(532 \mathrm{~nm})$ or tripled $(366 \mathrm{~nm})$ and when used with an Optical Parametric Oscillator provide wavelengths that range from about $650 \mathrm{~nm}$ to $2,500 \mathrm{~nm}$ for a $532 \mathrm{~nm}$ pump and from about $400 \mathrm{~nm}$ to 2,600 nm for 355 $\mathrm{nm}$ pump. Several vendors provide such packages. However, the energy output of these lasers in the IR past say $2,000 \mathrm{~nm}$ is about 40 and $15 \mathrm{~mJ}$ per pulse for $532 \mathrm{~nm}$ and $355 \mathrm{~nm}$ pump radiation, respectively. At repetition rates of $10 \mathrm{~Hz}$ these yield powers below 1 watt. Another aspect of these types of laser/OPO package is that the linewidth of such lasers in the IR range above 2,000 $\mathrm{nm}$ are about 80 and $20 \mathrm{~cm}^{-1}$ for $532 \mathrm{~nm}$ and $355 \mathrm{~nm}$, respectively. Such broad linewidths for the OPO should be beneficial for resonant absorption as linewidths are fairly broad in polymers and organic materials making fine tuning of the exact laser wavelength not that critical. While the available output energies from these OPOs may be useful for deposition of some materials the output wavelengths below 3,000 $\mathrm{nm}$ put these lasers well outside the stretching vibrational bands of most $\mathrm{O}-\mathrm{H}$ molecules incorporated in organic materials. These are the bands that will likely provide most of the coupling for organic materials using RIRPLD. These lasers also have pulse lengths on the order of 3-5 ns. Thus, their use on solid polymer targets may be limited.

In spite of the lack of ideal lasers RIRPLD equipment has been built. Figure 9 shows a photo of RIRPLD tool that utilizes a Continuum PowerLite Nd:YAG laser and SureLite OPO. The system incorporates the ability to use either a MAPLE or a solid organic target as desired. Like a basic MAPLE system this system includes a turbo pump with argon MFC and closed-loop pressure control. The system includes a $2^{\prime \prime}$ diameter substrate holder along with a $300^{\circ} \mathrm{C}$ substrate heater and is fully computer controlled. Also included is a loadlock for transfer of substrates ranging in size up to $2^{\prime \prime}$ diameter. As mentioned above, loadlocks are well worth the added cost for MAPLE processes to significantly reduce the time needed to grow films.

One should be aware that such tunable lasers require more than just the laser for optimal operation. The user should plan on purchasing a good joule meter that operates over the wavelength and peak power region of interest as well as a wideband spectrometer for maintaining calibration of the OPO which can add significant cost to the overall system. For instance a spectrometer that covers the range from 2.0 to 4.5 microns costs about $\$ 30,000$.

Just like the MAPLE process both the RIRPLD and RIRPLD/MAPLE process still have several outstanding questions remaining about how to optimize these techniques. These include the following. 
1. What is the angular distribution of the plume produced in the RIRPLD process for solid or MAPLE targets? How does this depend on laser wavelength and pulse length and chamber background pressure?

2. What types of film thickness uniformity can be produced over large sample sizes?

3. What types of deposition rate can be achieved with current or future lasers?

4. How do films deposited by RIRPLD/MAPLE compare to those produced by direct RIRPLD from a solid target?

5. What is the best pulse length and wavelength to use for this process for given specific organic materials? Is one type of laser with enough tuning range sufficient to cover most materials of interest?

6. Are optical components (mirrors, windows, and lenses) available with broadband AR and HR coatings that will handle the expected high peak powers from new tunable lasers?

\section{Conclusions}

Both MAPLE and RIRPLD are unique processes for the deposition of polymer films and these processes are still being developed and new techniques explored. There is a need for laser development for both of these processes shifting to longer wavelengths, shorter pulse lengths, and lower photon energies to be more effective in terms of the quality of films deposited and the growth rates obtained. For the MAPLE process a pulsed laser with a fixed wavelength bit further in the IR ( 3.5 microns) with lower energy per photon and one with shorter pulse length than a Er:YAG laser would be clearly beneficial. Higher pulse energy and repetition rates would also lead to higher growth rates.

RIRPLD clearly has the potential to provide a means to easily deposit a range of organic materials spanning a wide array of applications as noted in other companion papers within this journal. A laser with a wide tuning range in the $\sim 2.5$ to $\sim 5.0$ micron band with output power in the range of $\sim 10-20$ watts would be very enabling for this process. New lasers under development may soon be available to meet some of these specifications. Many questions still remain about how to optimize both processes for organic thin-film growth and hopefully new developments will be realized in the near future to further enable both MAPLE and RIRPLD thin-film processing.

Open Access This article is distributed under the terms of the Creative Commons Attribution Noncommercial License which permits any noncommercial use, distribution, and reproduction in any medium, provided the original author(s) and source are credited.

\section{References}

1. D.B. Chrisey, G.K. Hubler, Pulsed Laser Deposition of Thin Films (Wiley-Interscience, New York, 1994)

2. R. Eason, Pulsed Laser Deposition of Thin Films; ApplicationsLED Growth of Functional Materials (Wiley-Interscience, New York, 2007)

3. A. Piqué, R.A. McGill, D.B. Chrisey, D. Leonhardt, T.E. Mslna, B.J. Spargo, J.H. Callahan, R.W. Vachet, R. Chung, M.A. Bucaro, Thin Solid Films 356, 536 (1999)

4. R.A. McGill, D.B. Chrisey, U.S. Patent, 6,025,036, February 15th, 2000

5. D.M. Bubb, J.H. Callahan, J.S. Horwitz, R.A. McGill, E.J. Houser, D.B. Chrisey, R.F. Haglund, M.R. Papantonakis, M. Galicia, A. Vertes, J. Vac. Sci. Technol., A 19m, 2698-2702 (2001)

6. A. Stiff-Roberts, Private communication, Duke University, January, 2011

7. J.A. Greer, T.E. Parker, M. Rothschild, D.J. Erlich, in Proceedings of the 41st Annual Symposium on Frequency Control (1987), pp. 351-359

8. R. Pate, A.D. Stiff-Roberts, Chem. Phys. Lett. 477, 406-410 (2009)

9. M. Duering, R. Haglund, L.-D. Barry, Resonant infrared pulsed laser ablation of polymers with single picosecond pulses generated by an optical parametric amplifier, CLEO Technical Digest, paper CMH3 (2010) 